UC-34c

Reporting Date: May 1975 Issued: July 1975

\title{
More 14-MeV, Neutron-Induced Gamma-Ray Production Cross Sections
}

by

\author{
Darrell M. Drake \\ Edward D. Arthur \\ Myron G. Silbert
}

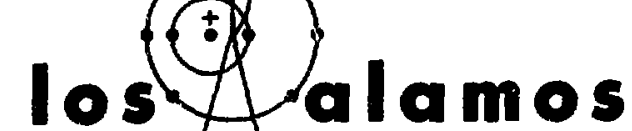

\section{-scisentifte daboratory}

of the University of Colifornia

LOS ALAMOS, NEW MEXICO 87545

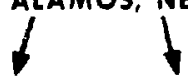

An Affirmative Action/Equal Opportunity Employer 
Printed in the United States of America. Available from National Technical Information Serrice

US Department of Commerce

5285 Port Royal Rood Springtield, VA 22151

Price: Printed Copy \$4.00 Microfiche $\$ 2.25$

This report was prepared as an account of work sponsored

bs the United States Government. Nejither the United States

nor the United States Enerky Research and bevelopment Ad-
miniatration, nor eny of their employees, nor any of their con-

tractors, subcontractors, or their employees, mpkes ony

warranty. express or implied, or assumes any legol liebility or

resp informetion eoperatus produrt. or process disclosed, or

any information, noperatus, prodvet. or procens disclosed, or
represents that its une would not infrisge privatejy owned
rikhts. 
MORE 14-MeV, NEUTRON-INDUCED GAMMA-RAY

\section{PRODUCTION CROSS SECTIONS}

by

Darrell M. Drake, Edward D. Arthur, and Myron G. Silbert
This repont was prepared as sponkined by the Prepared an an account of wort

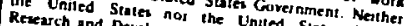

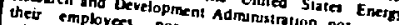
subcontractons, or nor int of thets con any ur

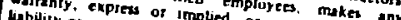

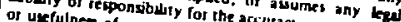
process dies of any injurmation apposs compleiteres tnfonge ponratedy, or represems that it us product of

ABSTRACT

A pulsed 14.2-MeV neutron source and $\mathrm{NaI}(\mathrm{Tl})$ gamma-ray spect rometer were used to measure gamma-ray production cross sections for beryllium, carbon, magnesium, aluminum, silicon, calcium, titanium, vanadium, chromium, iron, copper, niobium, molybdenum, thorium, and $238 \mathrm{U}$.

\section{INTRODUCTION}

Gamma-ray production cross sections were measured for samples of beryllium, carbon, magnesium, aluminum, silicon, calcium, titanium, vanadium, chromium, iron, copper, niovium, molybdenum, thorium, and ${ }^{238} \mathrm{U}$ that were bombarded with a pulsed $14.2 \mathrm{MeV}$ neutron beam obtained from the ${ }^{2} H(t, n){ }^{4}$ He reaction. $A$ first set of such measurements was reported in LA-5662-MS. Cross sections for five of the LA-5662-MS samples were measured several months later for the present report, and these data can be used as a check for consistency.

Data were taken at $90^{\circ}, 110^{\circ}$, and $130^{\circ}$ for most of the samples.

\section{EXPER IMENTAL ARRANGEMENT}

Figure 1 shows the experimental arrangement. A chopped beam of tritons (10-ns time width at $2-\mathrm{mHz}$ repetition rate) was accelerated to $2.3 \mathrm{MeV}$ by the Los Alamos Scientific Laboratory vertical Van de Graaff. The triton beam pulses were compressed to a time width of $1 \mathrm{~ns}$ by a Mobley bunching system and directed into a deuterium gas target. In this experiment, the triton bean was stopped in the deuterium gas, whereas in the arrangenent described in LA-5662-MS the tritons passed thruugh the gas and impinged on a gold beam stop with an energy of about $400 \mathrm{keV}$. When the beam is stopped in the deuterium gas, the number of neutrons per microampere approximately doubles, and the neutron energy spread is slightly increased.

Emitted neutrons, at $90^{\circ}$ to the triton beam and having a mean energy of $14.2 \mathrm{McV}$, interacted with one of the samples placed about $100 \mathrm{~mm}$ from the neut ren source. The energy spread of the neutrons intercepted by the samples was about $\pm 0.5 \mathrm{MeV}$. Gamna rays produced from the bombarded samples were collimated and pulse-height analyzed by a heavily shielded NaI(T1) crystal and photomultiplier system.

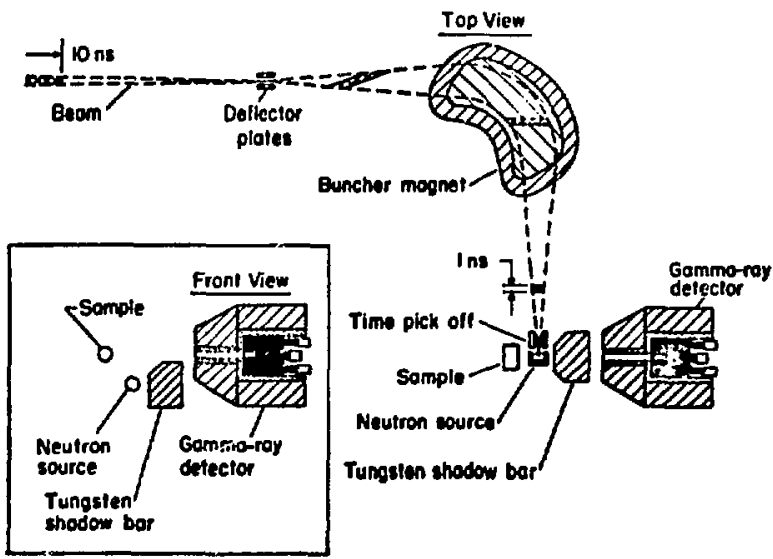

Fig. 1. Experimental arrangement for the measurements of gamma-ray spectra. The detector and sample are about $100 \mathrm{~mm}$ above the plane defined by the beam path. 
An anti-Compton NaI(T1) scintillator surrounding the center crystal was used to suppress further the background events and to improve the response functions. The pulsed neutron beam allowed time-of-tlight (TOF) discrimination by separating the desired gamma rays from neutron-related and other background events in the crystal.

Angles were changed by rotating the sample about the beam 1 ine and moving the gainma-ray spectrometer forward or backward to compensate for the change in sample-to-crystal distance.

\section{DATA RLDUCTIOA}

Procedures for background subtraction, responsc functions, flux measurements, multiple scattering, and the formula for cross-section calculations were the same as those given in Sec. 111 of LA-5662-MS. The technique used for estimation of errors is described in Sec, IV of that report.

\section{RESUTSS}

Cross sections are listed in Table I for gamnaray production by al! samples except beryllium and iarbon. In Table 1, cross sections are listed as millibarns per steradian in 100-keV gamma-ray energy intervals from 200 to $4000 \mathrm{keV}$, and in $500-\mathrm{keV}$ intervais above $4000 \mathrm{keV}$.

No gamma rays were observed from beryllium except for the $0.48-\mathrm{MeV}$ gamma-ray from ${ }^{9} \mathrm{Be}(n, t)^{7} \mathrm{Li}$, with a cross section of $0.7 \pm 0.2 \mathrm{mb} / \mathrm{sr}$. Table 11 lists observed cross sections for beryllium gamma rays from 0.5 to $5.0 \mathrm{MeV}$ in $0.5-\mathrm{MeV}$ intervals, along with corresponding standard deviations.

For the lighter elements (carbon through iron), cross sections for separable gallma rays are given in Table III. Some of these cross sections inight include contributions from nearby ganma rays. For example, the $0.85-\mathrm{MeV}$ ganna-ray cross section listed for iron irrobably contains the $0.93-M e V$ gamma rays from the ${ }^{56} \mathrm{Fe}(n, 2 \mathrm{n})^{55} \mathrm{Fe}$ reaction. Therc are some peaks in the spectra for heavier elements, but no at rempt was made to analyze them as single gamma rays.

Tabie IV lists the energy-weighted integral cross sections, $4 \pi \int \mathrm{L}_{\gamma} \mathrm{c}\left(\theta, \mathrm{E}_{\gamma}\right) \mathrm{dL} \mathrm{E}_{\gamma}$ from $\mathrm{E}_{\gamma}=0.3$ to 8.5 MeV, for all elements included in this report. 
TABLE I - DIFFERENTIAL GAMMA-RAY PRODUCTION CROSS SECTIONS AS A FUNCTION OF GAMMA-RAY ENERGY

\begin{tabular}{|c|c|c|c|c|c|c|c|c|c|c|c|c|c|}
\hline \multicolumn{2}{|c|}{ Element $\rightarrow$} & \multicolumn{6}{|c|}{ MAGNESIUM } & \multicolumn{6}{|c|}{ ALUMINUM } \\
\hline Ang le & $\rightarrow$ & 90 & & $110^{\circ}$ & & $130^{\circ}$ & & $90^{\circ}$ & & 110 & & 130 & \\
\hline \multicolumn{2}{|c|}{$\begin{array}{c}\text { Energy } \\
\text { Interval } \\
(\mathrm{MeV}) \\
\end{array}$} & $\begin{array}{l}\text { Cross } \\
\text { Section } \\
(\mathrm{mb} / \mathrm{sr}) \\
\end{array}$ & $\begin{array}{l}\text { Estimated } \\
\text { Uncer- } \\
\text { tainty } \\
\text { (mb/sr) } \\
\end{array}$ & $\begin{array}{l}\text { Cross } \\
\text { Section } \\
\text { (mb/sr) } \\
\end{array}$ & $\begin{array}{l}\text { Estimated } \\
\text { Uncer- } \\
\text { tainty } \\
\text { (mb/sr) } \\
\end{array}$ & $\begin{array}{l}\text { Cross } \\
\text { Section } \\
(\mathrm{mb} / \mathrm{sr}) \\
\end{array}$ & $\begin{array}{l}\text { Estimated } \\
\text { Uncer- } \\
\text { tainty } \\
\text { (mb/sr) }\end{array}$ & $\begin{array}{l}\text { Cross } \\
\text { Section } \\
(\mathrm{mb} / \mathrm{sr}) \\
\end{array}$ & $\begin{array}{l}\text { Estimated } \\
\text { Uncer- } \\
\text { tainty } \\
\text { (mb/sr) } \\
\end{array}$ & $\begin{array}{l}\text { Cross } \\
\text { Section } \\
(\mathrm{mb} / \mathrm{sr}) \\
\end{array}$ & $\begin{array}{l}\text { Estimated } \\
\text { Uncer- } \\
\text { tainty } \\
\text { (mb/sr) } \\
\end{array}$ & $\begin{array}{c}\text { Cross } \\
\text { Sect ion } \\
\text { (mb/sr) }\end{array}$ & $\begin{array}{l}\text { Estimated } \\
\text { Uncer- } \\
\text { tainty } \\
\text { (mb/sr) } \\
\end{array}$ \\
\hline $\begin{array}{l}0.7 \\
0.3 \\
0.4 \\
0.5 \\
0.4 \\
0.7 \\
0.4 \\
0.9 \\
1.0 \\
1.1 \\
1.2 \\
1.3 \\
1.4 \\
1.5 \\
1.5 \\
1.7 \\
1.8 \\
1.4\end{array}$ & $\begin{array}{l}0.3 \\
0.4 \\
0.5 \\
0.4 \\
0.7 \\
0.9 \\
0.9 \\
1.0 \\
1.1 \\
1.2 \\
1.3 \\
1.4 \\
1.5 \\
1.5 \\
1.7 \\
1.8 \\
1.9 \\
2.0\end{array}$ & $\begin{array}{l}9.1 \\
10.0 \\
4.3 \\
2.2 \\
1.03 \\
1.6 \\
2.8 \\
2.9 \\
2.3 \\
3.2 \\
4.3 \\
13.3 \\
10.9 \\
3.0 \\
2.3 \\
3.0 \\
3.9 \\
2.2\end{array}$ & $\begin{array}{l}3.3 \\
3.3 \\
0.0 \\
0.23 \\
0.12 \\
0.2 \\
0.3 \\
0.3 \\
0.3 \\
0.3 \\
0.4 \\
1.3 \\
1.1 \\
0.3 \\
0.2 \\
0.3 \\
0.4 \\
0.2\end{array}$ & $\begin{array}{l}6.4 \\
7.0 \\
3.4 \\
2.0 \\
1.06 \\
1.5 \\
2.7 \\
2.2 \\
2.2 \\
2.8 \\
4.7 \\
14.6 \\
10.0 \\
3.4 \\
2.1 \\
3.2 \\
3.5 \\
2.0\end{array}$ & $\begin{array}{l}2.3 \\
2.2 \\
0.7 \\
0.2 \\
0.17 \\
0.2 \\
0.3 \\
0.2 \\
0.2 \\
0.3 \\
0.5 \\
1.5 \\
1.0 \\
0.4 \\
0.2 \\
0.3 \\
0.4 \\
0.2\end{array}$ & $\begin{array}{l}6.1 \\
6.1 \\
3.9 \\
1.15 \\
0.91 \\
1.7 \\
2.8 \\
2.1 \\
2.1 \\
2.5 \\
7.1 \\
16.3 \\
8.1 \\
2.0 \\
2.1 \\
3.3 \\
3.7 \\
1.8\end{array}$ & $\begin{array}{l}2.1 \\
1.8 \\
0.8 \\
0.14 \\
0.11 \\
0.2 \\
0.3 \\
0.2 \\
0.3 \\
0.3 \\
0.7 \\
1.6 \\
0.8 \\
0.2 \\
0.2 \\
0.3 \\
0.4 \\
0.2\end{array}$ & $\begin{array}{l}6.9 \\
5.9 \\
4.3 \\
1.9 \\
1.09 \\
2.8 \\
4.4 \\
6.2 \\
5.5 \\
2.7 \\
1.8 \\
2.1 \\
2.2 \\
1.9 \\
2.8 \\
5.9 \\
7.3 \\
4.3\end{array}$ & $\begin{array}{l}0.1 \\
1.8 \\
0.9 \\
0.2 \\
0.12 \\
0.3 \\
0.4 \\
0.6 \\
0.6 \\
0.3 \\
0.2 \\
0.2 \\
0.7 \\
0.7 \\
0.3 \\
0.6 \\
0.7 \\
0.4\end{array}$ & $\begin{array}{l}4.1 \\
2.7 \\
3.2 \\
1.4 \\
1.01 \\
2.6 \\
3.7 \\
5.6 \\
5.0 \\
7.1 \\
1.5 \\
1.8 \\
1.9 \\
2.0 \\
2.6 \\
5.9 \\
5.6 \\
3.8\end{array}$ & $\begin{array}{l}1.2 \\
0.4 \\
0.0 \\
0.2 \\
0.11 \\
0.3 \\
0.4 \\
0.0 \\
0.5 \\
0.2 \\
0.2 \\
0.2 \\
0.2 \\
0.2 \\
0.3 \\
0.6 \\
0.7 \\
0.4\end{array}$ & $\begin{array}{l}3.0 \\
2.4 \\
3.1 \\
1.3 \\
0.71 \\
2.8 \\
4 . ? \\
5.5 \\
4.2 \\
2.1 \\
1.7 \\
1.7 \\
1.7 \\
1.9 \\
3.4 \\
6.8 \\
6.5 \\
3.4\end{array}$ & $\begin{array}{l}0.9 \\
0.14 \\
0.6 \\
0.7 \\
0.09 \\
0.3 \\
0.4 \\
0.6 \\
0.4 \\
0.7 \\
0 . ? \\
0 . ? \\
0.2 \\
0 . ? \\
0.3 \\
0.7 \\
0.7 \\
0.3\end{array}$ \\
\hline $\begin{array}{l}1.4 \\
? .0 \\
? .1 \\
3.0 \\
7.3 \\
7.4 \\
2.5 \\
2.5 \\
? .7 \\
3.4 \\
? .9 \\
\end{array}$ & $\begin{array}{l}2.0 \\
2.1 \\
2.2 \\
2.3 \\
2.4 \\
2.5 \\
2.5 \\
2.7 \\
2.8 \\
2.9 \\
3.0\end{array}$ & $\begin{array}{l}1.24 \\
0.94 \\
1.11 \\
1.00 \\
1.4 \\
1.22 \\
1.5 \\
1.6 \\
1.7 \\
1.11\end{array}$ & $\begin{array}{l}0.14 \\
0.11 \\
0.13 \\
0.12 \\
0.7 \\
0.14 \\
0.2 \\
0 . ? \\
0.2 \\
0.12\end{array}$ & $\begin{array}{c}-\frac{c}{1}: \frac{0}{25} \\
0.78 \\
1.21 \\
1.18 \\
1.22 \\
1.4 \\
1.6 \\
1.8 \\
1.5 \\
1.4\end{array}$ & $\begin{array}{l}0.0 \\
0.14 \\
0.09 \\
0.14 \\
0.13 \\
0.14 \\
0.2 \\
0.2 \\
0.2 \\
0.2 \\
0.2\end{array}$ & $\begin{array}{l}1.01 \\
1.11 \\
1.03 \\
1.3 \\
1.7 \\
1.7 \\
2.0 \\
2.0 \\
1.7 \\
1.10\end{array}$ & $\begin{array}{l}0.6 \\
0.14 \\
0.13 \\
0.12 \\
0.1 \\
0.2 \\
0.2 \\
0.2 \\
0.2 \\
0.2 \\
0.13\end{array}$ & $\begin{array}{l}2.4 \\
3.7 \\
5.2 \\
3.9 \\
2.5 \\
1.9 \\
1.6 \\
1.3 \\
1.8 \\
2.9 \\
\end{array}$ & $\begin{array}{l}0.3 \\
0.4 \\
0.5 \\
0.4 \\
0.3 \\
0.3 \\
0.2 \\
0.1 \\
0.2 \\
0.3 \\
\end{array}$ & $\begin{array}{l}3.2 \\
4.0 \\
5.1 \\
3.6 \\
2.2 \\
1.7 \\
1.5 \\
1.4 \\
1.9 \\
3.1\end{array}$ & $\begin{array}{l}0.2 \\
0.4 \\
0.5 \\
0.4 \\
0.2 \\
0.2 \\
0.2 \\
0.1 \\
0.2 \\
0.3\end{array}$ & $\begin{array}{l}3.04 \\
4.9 \\
5.3 \\
3.1 \\
2.1 \\
1.7 \\
1.5 \\
1.4 \\
2.7 \\
3.5 \\
\end{array}$ & $\begin{array}{l}0.0 .3 \\
0.3 \\
0.5 \\
0.5 \\
0.3 \\
0.2 \\
0.2 \\
0.2 \\
0.2 \\
0.3 \\
0.4\end{array}$ \\
\hline $\begin{array}{l}3.0 \\
3.1 \\
3.7 \\
3.3 \\
3.4 \\
3.5 \\
3.6 \\
3.7 \\
3.4 \\
3.9 \\
\end{array}$ & $\begin{array}{l}3.1 \\
3.7 \\
3.3 \\
3.4 \\
3.5 \\
3.6 \\
3.7 \\
3.9 \\
3.9 \\
4.0\end{array}$ & $\begin{array}{l}0.95 \\
0.89 \\
0.77 \\
0.70 \\
0.68 \\
0.83 \\
0.79 \\
1.15 \\
1.04 \\
0.90\end{array}$ & $\begin{array}{l}0.11 \\
0.11 \\
0.10 \\
0.09 \\
0.09 \\
0.110 \\
0.10 \\
0.14 \\
0.113 \\
0.111\end{array}$ & $\begin{array}{l}0.93 \\
1.07 \\
0.75 \\
0.68 \\
0.67 \\
0.83 \\
0.90 \\
1.01 \\
1.15 \\
0.98\end{array}$ & $\begin{array}{l}0.11 \\
0.13 \\
0.09 \\
0.04 \\
0.09 \\
0.10 \\
0.11 \\
0.17 \\
0.14 \\
0.17\end{array}$ & $\begin{array}{l}0.85 \\
0.75 \\
0.87 \\
0.80 \\
0.97 \\
0.91 \\
1.17 \\
1.14 \\
0.94 \\
1.38\end{array}$ & $\begin{array}{l}0.11 \\
0.10 \\
0.110 \\
0.10 \\
0.111 \\
0.11 \\
0.11 \\
0.11 \\
0.12 \\
0.16\end{array}$ & $\begin{array}{l}3.2 \\
2.2 \\
1.5 \\
0.94 \\
0.83 \\
0.84 \\
0.79 \\
0.94 \\
0.91 \\
0.77\end{array}$ & $\begin{array}{l}0.3 \\
0.2 \\
0.2 \\
0.11 \\
0.10 \\
0.10 \\
0.09 \\
0.11 \\
0.10 \\
0.09\end{array}$ & $\begin{array}{l}2.9 \\
2.1 \\
1.26 \\
0.86 \\
0.75 \\
0.67 \\
0.70 \\
0.80 \\
0.88 \\
0.76\end{array}$ & $\begin{array}{l}0.3 \\
0.2 \\
0.14 \\
0.10 \\
0.04 \\
0.04 \\
0.04 \\
0.04 \\
0.10 \\
0.04\end{array}$ & $\begin{array}{l}2.8 \\
1.8 \\
1.11 \\
0.87 \\
0.82 \\
0.74 \\
0.84 \\
0.8 n \\
0.80 \\
1.0 ?\end{array}$ & $\begin{array}{l}0.3 \\
0.2 \\
0.12 \\
0.10 \\
0.10 \\
0.09 \\
0.10 \\
0.09 \\
0.10 \\
0.12\end{array}$ \\
\hline $\begin{array}{l}4.7 \\
4.5 \\
5.0 \\
5.5 \\
6.01 \\
6.5 \\
7.0 \\
7.5 \\
8.0\end{array}$ & $\begin{array}{l}4.5 \\
5.0 \\
5.5 \\
6.0 \\
6.5 \\
7.0 \\
7.5 \\
8.0 \\
8.5\end{array}$ & $\begin{array}{l}1.00 \\
0.79 \\
0.56 \\
0.61 \\
0.46 \\
0.55 \\
0.58 \\
0.41 \\
0.40\end{array}$ & $\begin{array}{l}0.13 \\
0.11 \\
0.09 \\
0.09 \\
0.08 \\
0.09 \\
0.10 \\
0.04 \\
0.08\end{array}$ & $\begin{array}{l}1.12 \\
0.82 \\
0.54 \\
0.67 \\
0.56 \\
0.58 \\
0.49 \\
0.38 \\
0.42\end{array}$ & $\begin{array}{l}0.14 \\
0.11 \\
0.08 \\
0.10 \\
0.09 \\
0.10 \\
0.09 \\
0.04 \\
0.04\end{array}$ & $\begin{array}{l}1.16 \\
0.72 \\
0.51 \\
0.80 \\
0.61 \\
0.58 \\
0.54 \\
0.45 \\
0.41\end{array}$ & $\begin{array}{l}0.15 \\
0.10 \\
0.08 \\
0.12 \\
0.10 \\
0.10 \\
0.10 \\
0.09 \\
0.09\end{array}$ & $\begin{array}{l}0.90 \\
0.86 \\
0.74 \\
0.52 \\
0.57 \\
0.49 \\
0.48 \\
0.39 \\
0.32\end{array}$ & $\begin{array}{l}0.11 \\
0.11 \\
0.10 \\
0.08 \\
0.09 \\
0.08 \\
0.08 \\
0.07 \\
0.06\end{array}$ & $\begin{array}{l}0.81 \\
0.83 \\
0.73 \\
0.50 \\
0.60 \\
0.41 \\
0.47 \\
0.33 \\
0.28\end{array}$ & $\begin{array}{l}0.10 \\
0.10 \\
0.10 \\
0.07 \\
0.04 \\
0.04 \\
0.04 \\
0.04 \\
0.56\end{array}$ & $\begin{array}{l}0.80 \\
0.79 \\
0.85 \\
0.24 \\
0.61 \\
0.50 \\
0.45 \\
0.37 \\
0.27\end{array}$ & $\begin{array}{l}0.11 \\
0.10 \\
0.09 \\
0.08 \\
0.09 \\
0.08 \\
0.08 \\
0.016 \\
0.06\end{array}$ \\
\hline
\end{tabular}




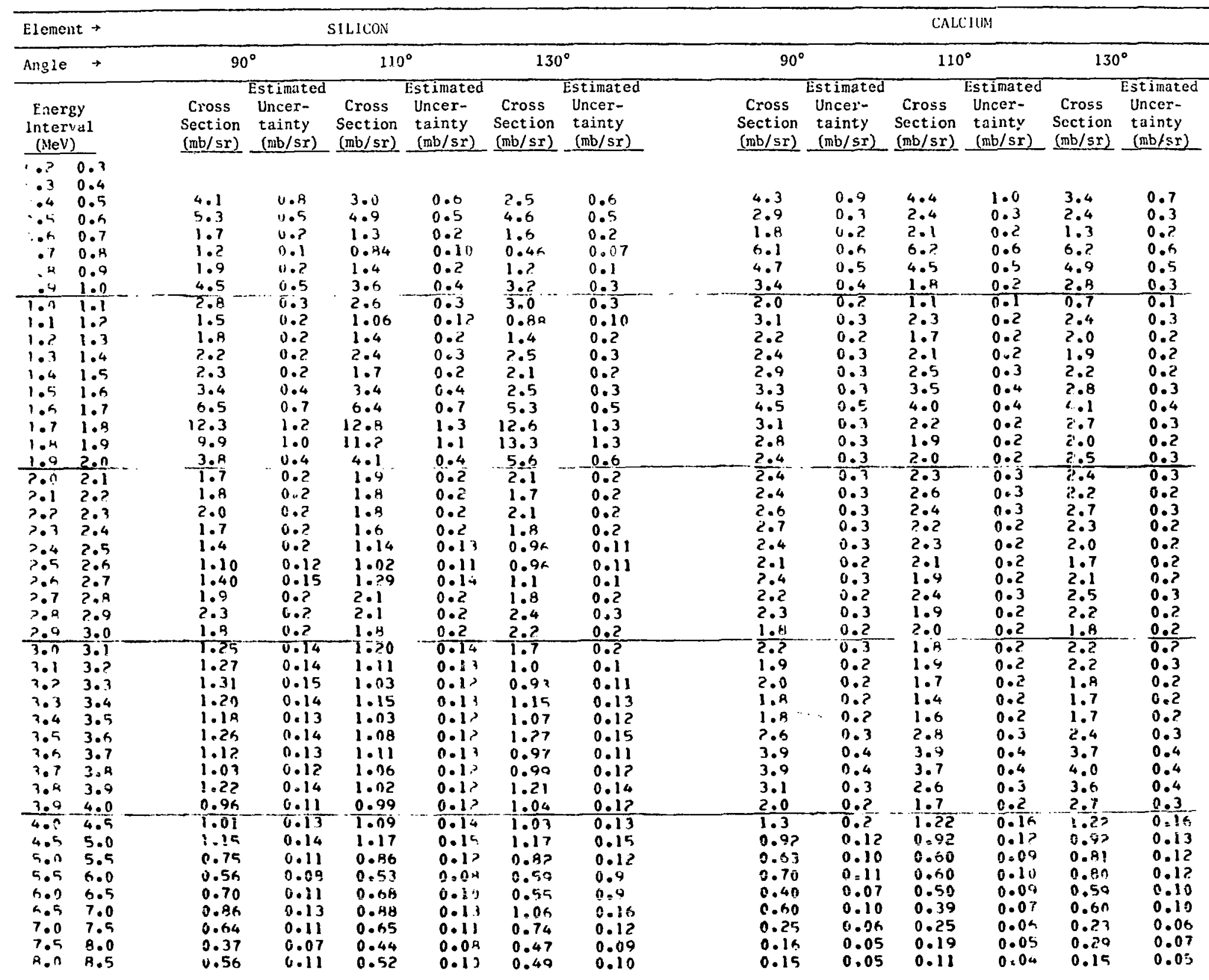




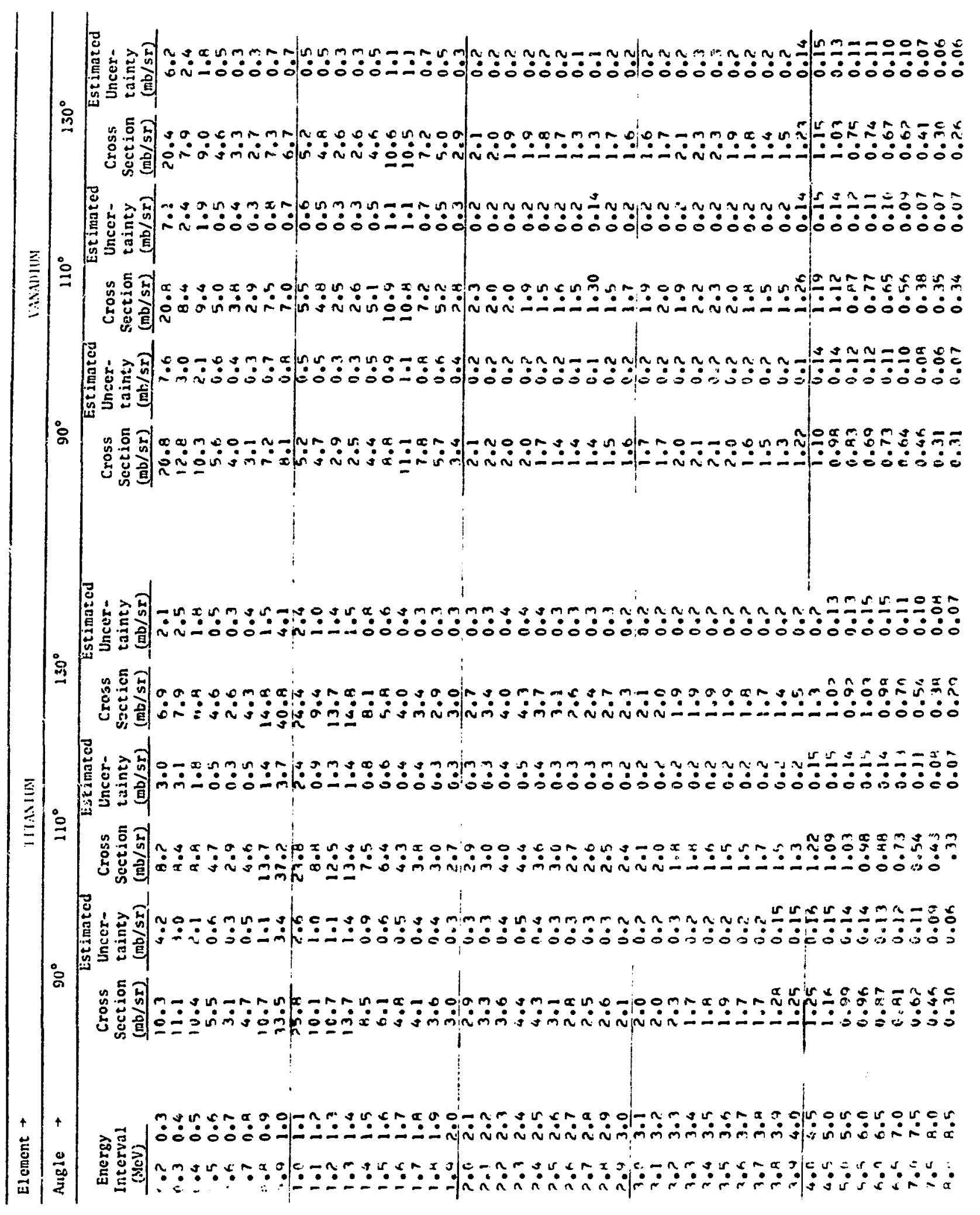




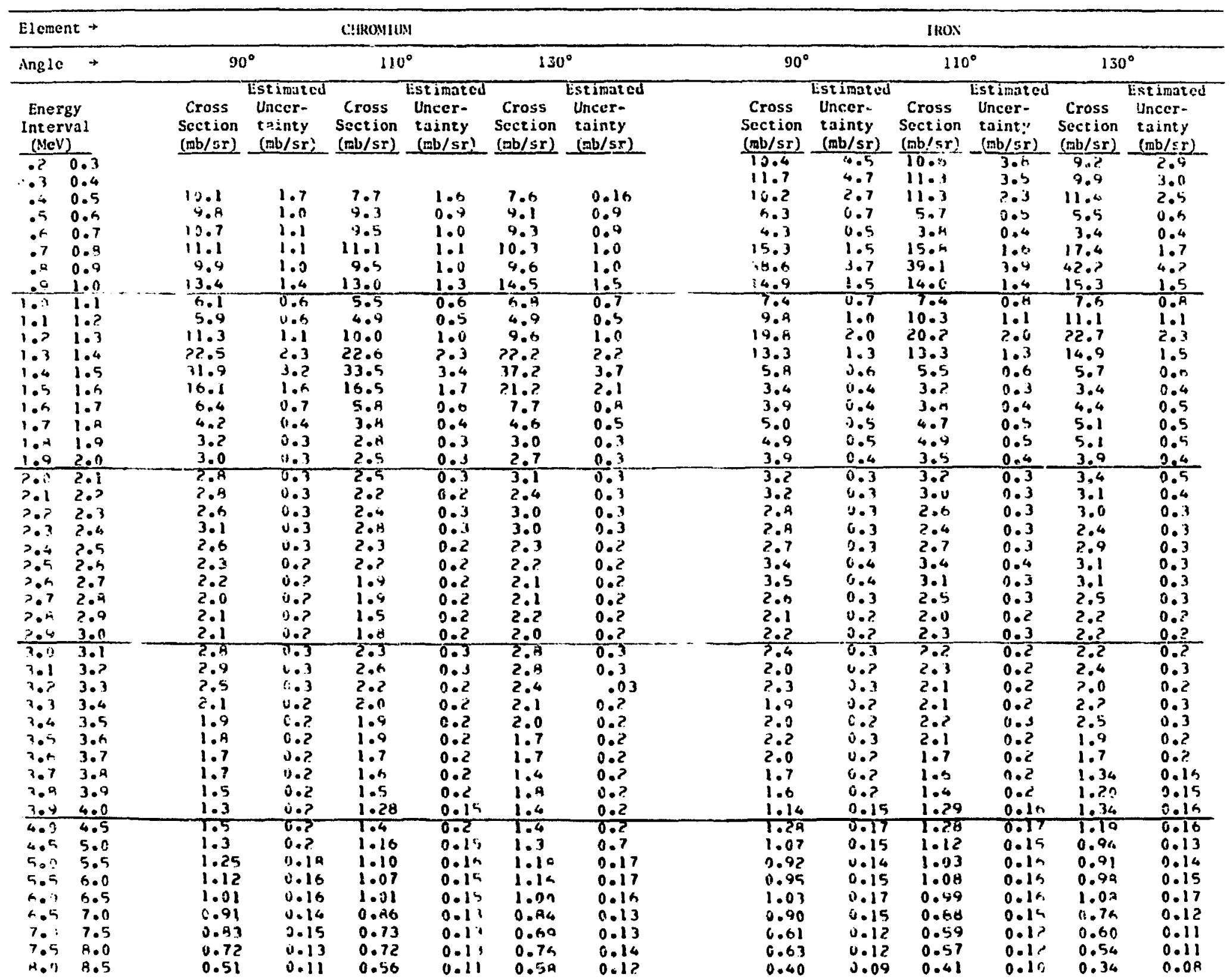




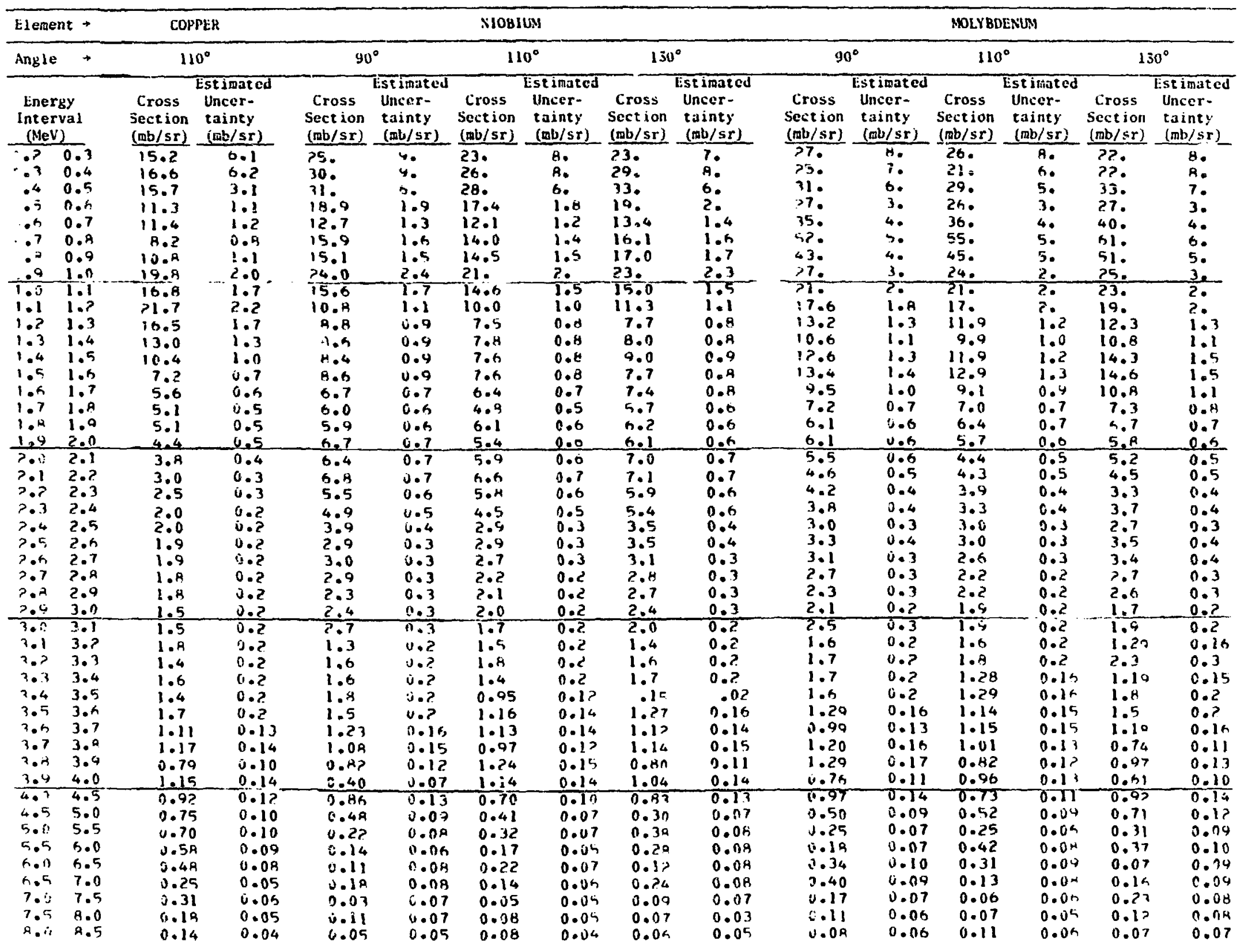




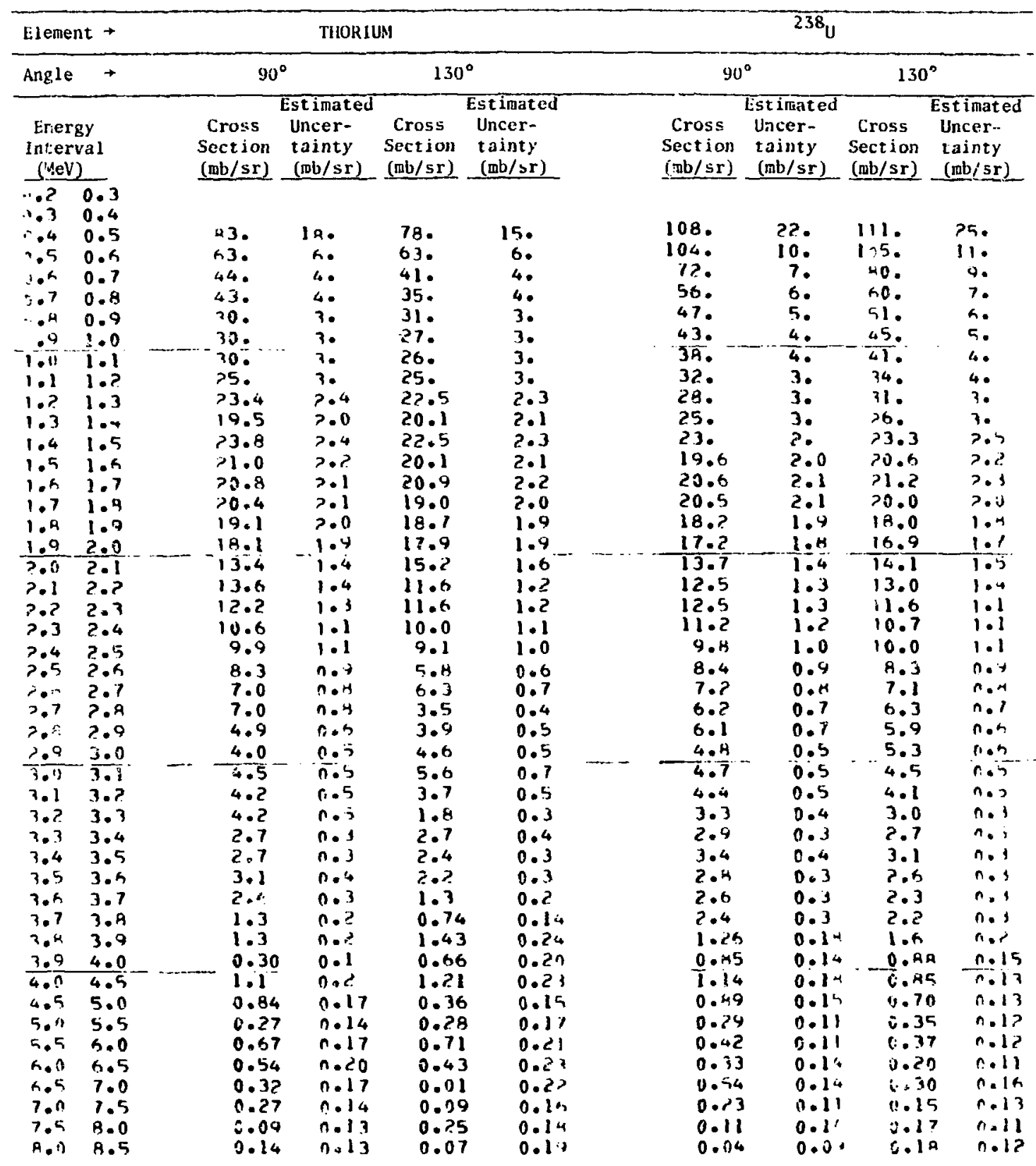


TABI.I: II

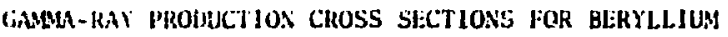
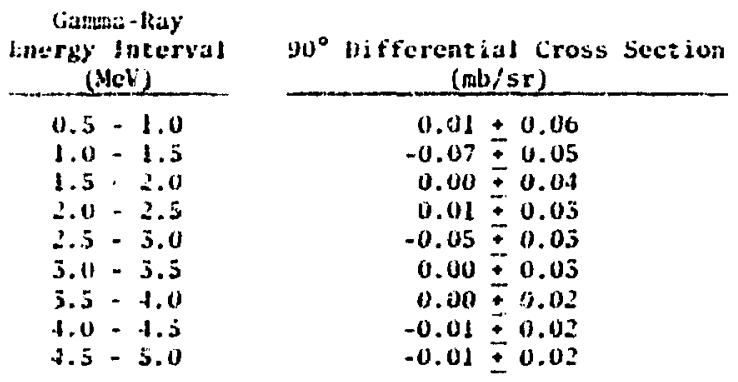

TRBL: III

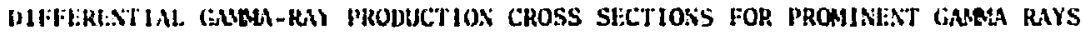

\begin{tabular}{|c|c|c|c|c|c|c|c|c|}
\hline \multirow[b]{2}{*}{ Llenent } & \multirow{2}{*}{\multicolumn{3}{|c|}{$\begin{array}{l}\text { lianna-Roy linergy } \\
\text { (HeV) }\end{array}$}} & \multicolumn{5}{|c|}{$\Delta$ (u) $(7 b / s r)$} \\
\hline & & & & \multicolumn{2}{|c|}{$\theta=a 0^{\circ}$} & \multicolumn{3}{|c|}{$j=110^{\circ}$} \\
\hline Carbon & \multicolumn{3}{|c|}{4.1} & 10.7 & \pm 1.5 & & \multicolumn{2}{|c|}{$13.6 \pm 1.9$} \\
\hline Magnosium & \multicolumn{3}{|c|}{1.37} & 22.0 & \pm 2.6 & & \multicolumn{2}{|c|}{$24.5 \pm 2.4$} \\
\hline Ml turimum & \multicolumn{3}{|c|}{$\begin{array}{c}0.5 .1+1.01 \\
1.8 \\
2.2 \\
5.0\end{array}$} & $\begin{array}{r}1.5 .8 \\
11.8 \\
9.8 \\
0.5\end{array}$ & $\begin{array}{l} \pm 1.5 \\
\pm 1.5 \\
\pm 1.5 \\
\pm 0.3\end{array}$ & & \multicolumn{2}{|c|}{$\begin{array}{r}11.5 \div 1.3 \\
10.7 \pm 1.3 \\
8.8 \div 1.1 \\
0.5 \pm 0.8\end{array}$} \\
\hline Silicon & \multicolumn{3}{|c|}{1. is } & 27.5 & \pm 5 & & \multicolumn{2}{|c|}{$29.9 \pm 5.0$} \\
\hline Tizani una & \multicolumn{3}{|c|}{$\begin{array}{l}0.99 \\
1.31\end{array}$} & $\begin{array}{l}04.8 \\
19.0\end{array}$ & $\pm \because .7$ & & \multicolumn{2}{|c|}{$\begin{array}{l}70.0 \pm 5.4 \\
22.2 \pm 2.5\end{array}$} \\
\hline Vanadjun & \multicolumn{3}{|c|}{1.00} & 16.2 & \pm 2.5 & & \multicolumn{2}{|c|}{$20.0 \pm 2.7$} \\
\hline chromium & \multicolumn{3}{|c|}{$1.5 .5+1 . .85$} & (19). & -3.1 & & \multicolumn{2}{|c|}{$74.0 \pm 8.6$} \\
\hline \multirow[t]{7}{*}{ Iron } & \multicolumn{3}{|c|}{$\begin{array}{l}0.85 \\
1.24\end{array}$} & $\begin{array}{l}51.5 \\
28.6\end{array}$ & \pm 6.2 & & \multicolumn{2}{|c|}{$\begin{array}{l}52.7 \pm 0.3 \\
29.5 \pm 5.6\end{array}$} \\
\hline & \multicolumn{8}{|c|}{ TABLI: IV } \\
\hline & \multicolumn{8}{|c|}{ 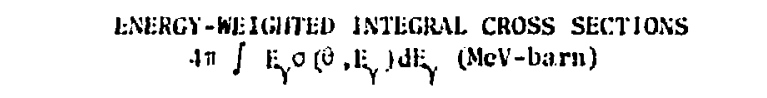 } \\
\hline & $\mathrm{J}$ : lement & 90 & & & & $10^{\circ}$ & & $30^{\circ}$ \\
\hline & $\begin{array}{l}\text { Beryllium } \\
\text { Carbon } \\
\text { Magnesium } \\
\text { Aluminum } \\
\text { Silicon } \\
\text { Calcium }\end{array}$ & $\begin{array}{l}0.05 \\
0.59 \\
3.9 \\
4.5 \\
4.7 \\
4.4\end{array}$ & $\frac{ \pm}{ \pm}$ & $\begin{array}{l}0.08 \\
0.4 \\
0.4 \\
0.5 \\
0.4\end{array}$ & $\begin{array}{l}0.82 \\
5.9 \\
4.0 \\
4.7 \\
4.0\end{array}$ & $\begin{array}{l} \pm 0.11 \\
\pm 0.4 \\
\pm 0.4 \\
\pm 0.5 \\
\pm 0.4\end{array}$ & $\begin{array}{l}1.07 \\
4.1 \\
4.1 \\
4.8 \\
4.5\end{array}$ & $\begin{array}{l} \pm 0.15 \\
\pm 0.4 \\
\pm 0.4 \\
\pm 0.5 \\
\pm 0.4\end{array}$ \\
\hline & $\begin{array}{l}\text { Titanium } \\
\text { Vanadium } \\
\text { Chromium } \\
\text { Jron }\end{array}$ & $\begin{array}{l}0.7 \\
5.0 \\
7.7 \\
6.5\end{array}$ & $\frac{ \pm}{ \pm}$ & $\begin{array}{l}0.7 \\
0.5 \\
0.7 \\
0.6\end{array}$ & $\begin{array}{l}\because .6 \\
5.1 \\
7.2 \\
6.8\end{array}$ & $\begin{array}{l} \pm 0.6 \\
\pm 0.5 \\
\pm 0.7 \\
\pm 0.7\end{array}$ & $\begin{array}{l}0.7 \\
4.9 \\
7.7 \\
0.8\end{array}$ & $\begin{array}{l} \pm 0.6 \\
\pm 0.5 \\
\pm 0.7 \\
\pm 0.7\end{array}$ \\
\hline & $\begin{array}{l}\text { Copper } \\
\text { Nolybdenum } \\
\text { Niobium } \\
\text { Thorium } \\
238 \mathrm{U}\end{array}$ & $\begin{array}{r}6.8 \\
5.3 \\
11.8 \\
13.2\end{array}$ & $\frac{ \pm}{ \pm}$ & $\begin{array}{l}0.7 \\
0.5 \\
1.2 \\
1.3\end{array}$ & $\begin{array}{l}5.4 \\
0.4 \\
4.9\end{array}$ & $\begin{array}{l} \pm 0.5 \\
\pm 0.7 \\
\pm 0.5\end{array}$ & $\begin{array}{r}7.0 \\
5.3 \\
12.8 \\
12.8\end{array}$ & $\begin{array}{l} \pm 0.7 \\
\pm 0.5 \\
\pm 1.3 \\
\pm 1.3\end{array}$ \\
\hline
\end{tabular}

